\title{
AUTOMORPHIC QUASIMEROMORPHIC MAPPINGS FOR TORSIONLESS HYPERBOLIC GROUPS
}

\author{
PEKKA TUKIA
}

A. Introduction. In this paper we consider groups of isometries of the hyperbolic space $H^{n}=\left\{\left(x_{1}, \ldots, x_{n}\right) \in R^{n}: x_{n}>0\right\}$ whose hyperbolic metric $d$ is given by $|d x| / x_{n}$. Let $G$ be such a group of isometries acting discontinuously in $H^{n}$. Martio and Srebro [3] raised the question whether there always exists a $G$-automorphic quasimeromorphic mapping $f: H^{n} \rightarrow \bar{R}^{n}=R^{n} \cup\{\infty\}$. That is, $f$ satisfies

$$
f(g(x))=f(x)
$$

for all $x \in H^{n}$ and $g \in G$ and, moreover, $f$ is quasimeromorphic in the sense of Martio and Srebro $[3,1.1]$. In the theory of quasiregular mappings, this problem is the analogue of the question of the existence of complex analytic maps automorphic with respect to a Fuchsian group.

We prove here the existence of such maps under the assumption that the group $G$ is torsionless and does not contain orientation reversing elements. More precisely, we prove

Theorem A. For every integer $n>1$ there are $K=K(n) \geqq 1$ and a positive integer $N=N(n)$ with the following property. Let $G$ be a discontinuous, torsionless group of isometries of $H^{n}$ not containing orientation reversing elements. Then there is a quasimeromorphic G-automorphic mapping $f: H^{n} \rightarrow \bar{R}^{n}$. Moreover, $f$ is $K$-quasimeromorphic and the local topological index $i(x, f)$ of $f$ is less than $N$ for every $x \in H^{n}$.

The local topological index $i(x, f)$ of $f$ at $x$ has been defined in $[3,2.6]$ and $f$ is $K$-quasimeromorphic if it is $\mathrm{ACL}^{n}$ and satisfies almost everywhere in $H^{n}$ the equation (1) of $[3,1.1]$.

We will prove Theorem A in the course of this paper and the proof is concluded in Section F. We remark that $G$ is torsionless if and only if it acts without fixed points in $H^{n}$, i.e. the equation $g(x)=x$ for some $x$ implies $g=\mathrm{id}$. The existence of a $G$-automorphic mapping $f: H^{n} \rightarrow \bar{R}^{n}$ implies that $G$ cannot contain orientation reversing elements. However, the assumption that $G$ is torsionless is unnecessarily strong. In fact, if the hyperbolic measure of $H^{n} / G$ is finite, and every $g \in G$ is orientation preserving, then it is known that there are quasimeromorphic $G$-automorphic mappings (cf. Martio and Srebro [4]). 
Our method is an extension of the method of Alexander [1] by which one can construct quasimeromorphic maps if $M=H^{n} / G$ is compact. A $G$-automorphic map can be identified with a map $M \rightarrow \bar{R}^{n}$. One first triangulates $M$ (in a sufficiently regular manner); let the set of vertices be $v_{1}, \ldots, v_{k}$. Next one finds points $b_{1}, \ldots, b_{k} \in$ $R^{n}$ in general position. One then maps an $n$-simplex $T$ of $M$ with vertices $v_{i_{0}}, \ldots, v_{i_{n}}$, $i_{0}<\ldots<i_{n}$, either to the simplex $\Delta$ of $R^{n}$ with vertices $b_{i_{0}}, \ldots, b_{i_{n}}$ or to the outside of $\Delta$ in $\bar{R}^{n}$, depending on the orientations of $T$ and $\Delta$. The resulting map is quasimeromorphic. If $M$ is non-compact, one can construct by this method branched coverings $M \rightarrow \bar{R}^{n}$ but they are not in general quasimeromorphic. The gist of our method is that one can find such triangulations of $M$ which allow the construction of quasimeromorphic mappings by Alexander's method also if $M$ is non-compact. To find such triangulations, we construct in Section C triangulations of $H^{n}$ whose set of vertices is a beforehand given discrete set $A$. If this set $A$ is judiciously chosen, we get a $G$-invariant triangulation of $H^{n}$ (Theorem D) whose simplexes are not very flat. This makes it possible to apply Alexander's method in the construction of the quasimeromorphic map.

We have constructed such triangulations in the hyperbolic space. We could have done it as well in the euclidean space and since in a smooth Riemannian manifold every point has neighbourhoods whose metric is arbitrarily close to the euclidean, it seems possible to construct such triangulations for any smooth Riemannian manifold. Thus Theorem A should be valid in fact for any smooth Riemannian manifold, and not only for hyperbolic ones. Perhaps we can return to it.

Notation and terminology. Hyperbolic isometries of $H^{n}$ are also called Möbius transformations and groups of isometries Möbius groups. We use the words $G$-automorphic and $G$-invariant synonymously for a map $f$ defined in a $G$-invariant subset $A$ of $H^{n}$ (i.e. $g A=A$ for every $g \in G$ ) such that (A1) is true for every $x \in A$ and $g \in G$.

We use $d$ for the hyperbolic metric of $H^{n}$ and the euclidean distance of two points of $R^{n}$ is $|x-y|$. We denote by $d(A)$ and by $d(x, A)$ both the hyperbolic and euclidean diameter of a set and the distance of a point to a set, respecively. If confusion is possible, we say which metric we mean. The closed hyperbolic ball with center $x$ and radius $r$ is $D(x, r)$. The standard basis of $R^{n}$ is $e_{1}, \ldots, e_{n}$.

Interior, closure and boundary are usually taken in $H^{n}$ and are denoted int, cl and bd; the last one sometimes also $\partial$. If they are taken in some other space, we use a subscript, e.g. $\partial_{T}$.

We denote the identity map of a space by id.

B. Hyperbolic $k$-cells. In the next section we will construct special tessellations of $H^{n}$. For this we need to know what one means by a $k$-cell of $H^{n}$. We will now define these cells and prove what we need to know about them.

We mean by a convex subset of $H^{n}$ a set which is convex with respect to the 
hyperbolic metric. If $X \subset H^{n}$, we let

$$
\operatorname{Co}(X)
$$

denote the smallest closed and convex subset of $H^{n}$ such that $X \subset \operatorname{Co}(X)$. We say that $H$ is a half-space of $H^{n}$ if $H$ is the closure of a component of $H^{n} \backslash T$ for some hyperbolic $(n-1)$-subplane of $H^{n}$; similarly one defines half-spaces of hyperbolic $i$-subplanes of $H^{n}$. Then we have

Lemma B1. If $T$ is a hyperbolic subplane of $H^{n}$ and $X \subset T$, then

$$
\operatorname{Co}(X)=\cap H
$$

where the intersection is taken over all such half-spaces $H$ of $T$ for which $H \supset X$ (and $\operatorname{Co}(X)=T$ if there are no such half-spaces).

Furthermore, if $x \in \mathrm{bd}_{T} \mathrm{Co}(X)$, then there is a half-space $H$ of $T$ such that $H \supset \mathrm{Co}(X)$ and $x \in \partial_{T} H$.

Proof. To prove the validity of (B2), denote the right-hand side of (B2) by $Z$. Then obviously $Z \supset \operatorname{Co}(X)$ since half-spaces are closed and convex. To prove the reverse inclusion, we find for every $x \in T \backslash \mathrm{Co}(X)$ a half-space $H$ such that $x \notin H \supset \operatorname{Co}(X)$. If $x \in T \backslash \operatorname{Co}(X)$, let $y \in \operatorname{Co}(X)$ be a point such that $d(x, y)=$ $d(x, \operatorname{Co}(X))$. Let $L$ be the hyperbolic line passing through $x$ and $y$. Let $H$ be the half-space such that $\partial H$ intersects $L$ orthogonally at $y$ and that $x \notin H$. We claim that $\operatorname{Co}(X) \subset H$. If this is not the case, pick a point $z \in \operatorname{Co}(X) \backslash H$. Let $S$ be the open hyperbolic line segment with endpoints $z$ and $y$. Then a point $y^{\prime}$ of $S$ near $y$ satisfies $d\left(y^{\prime}, x\right)<d(y, x)$ as is geometrically evident and can be proved precisely using hyperbolic trigonometry (see Beardon $[2,7.11]$ ). This is a contradiction since $S \subset \operatorname{Co}(X)$ and hence $d(y, x)=d(x, \operatorname{Co}(X)) \leqq d\left(y^{\prime}, x\right)$. Thus (B2) is true.

To get the second part of the lemma, pick $x \in \operatorname{bd}_{T} \operatorname{Co}(X)$. By the first part of the lemma, we can find a sequence $H_{i} \supset \mathrm{Co}(X)$ of half-spaces of $T$ such that there are $x_{i} \in \partial H_{i}$ for which $x_{i} \rightarrow x$ as $i \rightarrow \infty$. Then we can pass to a subsequence in such a way that $H_{i} \rightarrow H$ for some half-space $H$ (the limit being taken in an obvious topology). Then $H \supset \mathrm{Co}(X)$ and $x \in \partial H$ and the lemma is proved.

We can now define that a $k$-cell of $H^{n}$ is a set $C$ which is of the form

$$
C=\mathrm{Co}(V)
$$

for some finite, non-empty set $V \subset H^{n}$ such that the smallest hyperbolic subplane $T$ of $H^{n}$ containing $V$ has dimension $k$. We denote

$$
\text { int } C=\operatorname{int}_{T} C \text { and } \partial C=\operatorname{bd}_{T} C \text {. }
$$

We then define that a $p$-face (or a face) of a $k$-cell $C=\operatorname{Co}(V)$ as follows. There are no $p$-faces for $p<0$ or $p>k$. If $p=k$, then $F$ is a $p$-face of $C$ if and only if $F=C$. If $p=k-1$, then $F$ is a $p$-face of $C$ if $F$ is a maximal $p$-cell of the form

$$
F=\mathrm{Co}(U)
$$


where $U \subset V$ and such that $F \cap \operatorname{int} C=\emptyset$. For $p<k-1$ the definition is recursive: now $F$ is a $p$-face of $C$ if and only if $F$ is a $p$-face of some $(p+1)$-face of $C$. Obviously, a face of a face of $C$ is again a face of $C$. The 0 -faces of $C$ are also called the vertices of $C$.

We now give what we need to know of cells of $H^{n}$ in

Lemma B2. Let $C=\mathrm{Co}(V), V \subset H^{n}$ finite, be a $k$-cell of $H^{n}$. Then

(a) if $H$ is a half-space of $H^{n}$ such that $H \supset V$, then $H \supset C$ and

$$
\partial H \cap C=\operatorname{Co}(\partial H \cap V)
$$

and $\partial H \cap C$ is a face of $C$ if non-empty,

(b) if $x \in C$, then there is a face $F$ of $C$ such that $x \in \operatorname{int} F$.

Proof. If $H \supset V$, then obviously $H \supset C$. We then prove (B3). Note first that obviously $\partial H \cap C=\partial H \cap \mathrm{Co}(V) \supset \mathrm{Co}(\partial H \cap V)$. To get the opposite inequality, choose $x \in \partial H \backslash \operatorname{Co}(\partial H \cap V)$. Then by (B2) there is a half-space $H^{\prime}$ of $\partial H$ such that $H^{\prime} \supset \partial H \cap V$ and that $x \notin H^{\prime}$. Since $V$ is finite, we can find a half-space $H^{\prime \prime}$ of $H^{n}$ such that $H^{\prime \prime} \supset V$ and that $H^{\prime \prime} \cap H=H^{\prime}$. Then $H^{\prime \prime} \supset \mathrm{Co}(V)=C$ and $x \notin H^{\prime \prime}$. This implies (B3).

Suppose then that $F=\partial H \cap C \neq \emptyset$. We must show that $F$ is a face of $C$. In any case $F=\mathrm{Co}(\partial H \cap V)$ is a $p$-cell for some $0 \leqq p \leqq k$. If $p=k$, then $\partial H \supset C$ and hence this case is clear. If $p=k-1$, then obviously $F \cap \operatorname{int} C=\emptyset$ and if $U$ is a set such that $V \supset U \supset \partial H \cap V$, then $\operatorname{Co}(U) \cap$ int $C=\emptyset$ if and only if $U=$ $\partial H \cap V$. This proves that $F$ is a $(k-1)$-face of $C$.

Suppose now that $p<k-1$. We show that there is then a half-space $H^{\prime}$ of $H^{n}$ such that $H^{\prime} \supset C$ and that $\partial H^{\prime} \cap C$ is a $q$-cell where $p<q<k$. This gives then a recursive argument proving (a).

To see this let $T$ be the hyperbolic $k$-plane containing $C$. Then $T \cap \partial H$ is a $(k-1)$-plane and there is a $(k-2)$-plane $T^{\prime}$ in $T \cap \partial H$ which contains $\partial H \cap C$. If $a \in V \backslash \partial H$, let $L_{a}$ be the hyperbolic line passing through $a$ and intersecting $T^{\prime}$ orthogonally (if $T^{\prime}$ is a point, let $L_{a}$ be the line containing $\left.\{a\} \cup T^{\prime}\right)$. Let $\varphi_{a} \in(0, \pi / 2$ ] be the angle between $L_{a}$ and $\partial H$. Choose $a \in V \backslash \partial H$ such that $\varphi_{a}$ is minimal. By the minimality of $\varphi_{a}$ there is a half-space $H^{\prime \prime}$ of $T$ such that $H^{\prime \prime} \supset V$ and that $\partial H^{\prime \prime} \supset$ $T^{\prime} \cup\{a\}$. Note that $\partial H^{\prime \prime} \cap V$ is a proper subset of $V$. Now let $H^{\prime}$ be any half-space of $H^{n}$ such that $H^{\prime} \cap T=H^{\prime \prime}$. Then $H^{\prime} \supset V$ and $\partial H^{\prime} \cap C$ is a $q$-cell for some $q \in(p, k)$.

This proves (a). In case (b), let $T$ be as above the smallest hyperbolic subplane of $H^{n}$ containing $C$. If $x \in \operatorname{int} C$, then (b) is true for $F=C$. Otherwise, $x \in \partial C$ and now Lemma B1 implies that there is a half-space $H$ of $T$ such that $H \supset C$ and $x \in \partial H$. Then case (a) implies that $F_{1}=\partial H \cap C$ is a face of $C$ of dimension less than $k$. If $x \in$ int $F_{1}$, we are ready. Otherwise we repeat the above process and find a face $F_{2}$ or $F_{1}, F_{2} \neq F_{1}$, such that $x \in F_{2}$. After a finite number of steps we find a face $F$ of $C$ such that $x \in \operatorname{int} F$. 
C. Triangulations of $H^{n}$. Our proof of Theorem $\mathrm{A}$ is based on special triangulations of $H^{n+1}$ which we now construct. If $A$ is a discrete subset of $H^{n+1}$ satisfying a boundedness condition, then we prove that there is a triangulation of $H^{n+1}$ whose set of vertices is $A$. The construction of such a triangulation is based on a method which is fundamentally a construction dual to the familiar construction of fundamental domains for discrete groups of isometries of $H^{n}$ when $A=G x$ for some $x \in H^{n}$ and the $n$-cells are

$$
F_{a}=\left\{x \in H^{n}: d(x, a) \leqq d(x, b) \text { for } b \in A\right\},
$$

$a \in A$. However, our construction is direct and not based on the fact that $\left\{F_{a}\right\}_{a \in A}$ is a tessellation of $H^{n}$.

We have already constructed triangulations of this kind if $n=2$ (in a slightly more general setting) in [6, Section 4] but for the convenience of the reader we give the complete proof although many steps are directly parallel to those of [6].

Tessellations. Let $A \subset H^{n}$ be discrete. We first construct a tessellation of $H^{n}$ by $n$-cells, denoted $\mathscr{T}_{A}$, which covers $H^{n}$ under a mild condition on $A$. Later we will subdivide $\mathscr{T}_{A}$ into a triangulation of $H^{n}$.

The first step in the construction is to define the centers of the $n$-cells of $\mathscr{T}_{A}$; we denote this set by $V_{A}$. If we let $D_{v}$ be the closed hyperbolic disk with center $v$ and radius $d(v, A)$, then we can define that $v \in V_{A}$ if and only if

$$
A_{v}=D_{v} \cap A=\partial D_{v} \cap A
$$

is not contained in any proper hyperbolic subspace of $H^{n}$. It is not difficult to see that $V_{A}$ is discrete and obviously each $A_{v}$ is finite. Then, given $v \in V_{A}$, we define an $n$-cell $C_{v}$ of $H^{n}$ by

$$
C_{v}=\operatorname{Co}\left(A_{v}\right)
$$

where $\mathrm{Co}$ is as in (B1). Now we can define the family $\mathscr{T}_{A}$ of $n$-cells of $H^{n}$ by

$$
\mathscr{T}_{A}=\left\{C_{v}: v \in V_{A}\right\}
$$

This is the tessellation at which we have been aiming. Note that each $v \in V_{A}$ would be a vertex in the tessellation $\left\{F_{a}\right\}$ defined by $(\mathrm{Cl})$ and so our method is indeed dual to the construction of Dirichlet fundamental domains for discrete groups of $H^{n}$. That $\mathscr{T}_{A}$ is indeed a tessellation is shown by

Theorem C1. If $A$ is a discrete subset of $H^{n}$, then $\mathscr{T}_{A}$ is a tessellation in the sense that if $C, C^{\prime} \in \mathscr{T}_{A}$, then

$$
C \cap C^{\prime}
$$

is either empty or a common face.

Furthermore, suppose that there is $M>0$ such that

$$
d(x, A) \leqq M
$$


for all $x \in H^{n}$. Then $\mathscr{T}_{A}$ is a locally finite cover of $H^{n}$ such that for all $C \in \mathscr{T}_{A}$,

$$
d(C) \leqq 2 M .
$$

Proof. Let $C=C_{v}$ and $C^{\prime}=C_{v^{\prime}}$ where $v, v^{\prime} \in V_{A}$. We can assume that $v \neq v^{\prime}$. Since $C \subset D_{v}$ and $C^{\prime} \subset D_{v^{\prime}}, C \cap C^{\prime}=\emptyset$ if $D_{v} \cap D_{v^{\prime}}=\emptyset$. So we can assume that $D_{v} \cap D_{v^{\prime}} \neq \emptyset$. In this case let $T$ be the hyperbolic $n$-plane containing the $(n-2)$-circle $\partial D_{v} \cap \partial D_{v^{\prime}}$ (or, if $\partial D_{v} \cap \partial D_{v^{\prime}}=$ a point, let $T$ be the common tangent to $\partial D_{v}$ and $\left.\partial D_{v^{\prime}}\right)$. Let $H$ and $H^{\prime}$ be the two half-spaces of $H^{n}$ whose boundary is $T$. In view of (C2), we can name them in such a way that $A_{v} \subset H$ and $A_{v^{\prime}} \subset H^{\prime}$. Thus, by Lemma B2 (a), $C \subset H$ and $C^{\prime} \subset H^{\prime}$ and, since $A_{v} \cap A_{v^{\prime}}=A_{v} \cap T=A_{v^{\prime}} \cap T$,

$$
C \cap C^{\prime}=(C \cap T) \cap\left(C^{\prime} \cap T\right)=\operatorname{Co}\left(A_{v} \cap T\right) \cap \operatorname{Co}\left(A_{v^{\prime}} \cap T\right)=\operatorname{Co}\left(A_{v} \cap A_{v^{\prime}}\right)
$$

is a common face.

This proves the first paragraph. We then assume that (C5) is true for some $M>0$. We prove first (C6). This is an immediate consequence of (C2) and (C5). These imply that if $x \in A_{v}$, then $d(x, v) \leqq M$ and hence

$$
d\left(C_{v}\right) \leqq d\left(D_{v}\right) \leqq 2 M
$$

and (C6) is proved.

Next we prove that $\mathscr{T}_{A}$ is locally finite. Pick $x \in H^{n}$. If $C \in \mathscr{T}_{A}$ and $C \cap D(x, 1) \neq \emptyset$, then (C6) implies that $C \subset D(x, 2 M+1)$. Hence the vertices of $T$ are in the finite set $A \cap D(x, 2 M+1)$. It follows that the number of $C \in \mathscr{T}_{A}$ intersecting with $D(x, 1)$ is finite and this proves the local finiteness of $\mathscr{T}_{A}$.

We must now only prove that $\mathscr{T}_{A}$ covers $H^{n}$. Since every $T \in \mathscr{T}_{A}$ is closed and we now know that $\mathscr{T}_{A}$ is locally finite, it follows that $\cup \mathscr{T}_{A}$ is closed. As a consequence, to prove that $\mathscr{T}_{A}$ is a cover of $H^{n}$, it suffices to prove that (a) $\cup \mathscr{T}_{A} \neq \emptyset$ and (b) $\cup \mathscr{T}_{A}$ is open.

Now $\cup \mathscr{T}_{A} \neq \emptyset$ if and only if $\mathscr{T}_{A} \neq \emptyset$. We can prove that $\mathscr{T}_{A} \neq \emptyset$ as follows. Pick first $a_{0} \in A$. In view of (C5), there is such $a_{0}$. Let $R_{0}$ be a hyperbolic ray with endpoint $a_{0}$. Let $v_{0}^{\prime}$ be the first point on $R_{0}$ from $a_{0}$ such that there is $a_{1} \in A \backslash\left\{a_{0}\right\}$ with $d\left(v_{0}^{\prime}, a_{0}\right)=d\left(v_{0}^{\prime}, a_{1}\right)$. By (C5), there is such $a_{1}$.

Let now $T_{1}$ be the hyperbolic line containing $a_{0}$ and $a_{1}$ and let $v_{0}$ be the orthogonal projection of $v_{0}^{\prime}$ (in hyperbolic geometry) onto $T_{1}$. If we set $r_{0}=d\left(v_{0}, a_{0}\right)=$ $d\left(v_{0}, a_{1}\right)=d\left(v_{0}, A\right)>0$, then

$$
A_{1}=D\left(v_{0}, r_{0}\right) \cap A=\partial D\left(v_{0}, r_{0}\right) \cap A \supset\left\{a_{0}, a_{1}\right\} .
$$

We can now repeat the above process. Let $R_{1}$ be a hyperbolic ray with endpoint $v_{0}$ and which is orthogonal to $T_{1}$. Let $v_{1}^{\prime}$ be the first point on $R_{1}$ such that there is $a_{2} \in A \backslash A_{1}$ for which $d\left(v_{1}^{\prime}, a_{2}\right)=d\left(v_{1}^{\prime}, b\right)$ for $b \in A_{1}$. We now let $T_{2}$ be the hyperbolic $i$-plane $(i \geqq 2)$ containing $A_{1} \cup\left\{a_{2}\right\}$ and let $v_{1}$ be the orthogonal projection of $v_{1}^{\prime}$ onto $T_{2}$. It is clear that in this manner we finally find $v=v_{p} \in H^{n}, p \geqq 0$, a finite set $A_{v}=A_{p} \subset H^{n}$ which is not contained in any proper hyperbolic subplane of 
$H^{n}$ such that if $r=d(v, A)>0$, then

$$
A_{v}=D(v, r) \cap A=\partial D(v, r) \cap A .
$$

It follows that $v \in V_{A}$ and that $C_{v}=\operatorname{Co}\left(A_{v}\right) \in \mathscr{T}_{A} \neq \emptyset$.

The final step is to prove that $\cup \mathscr{T}_{A}$ is open. If $x \in \cup \mathscr{T}_{A}$, then by Lemma B2 (b), $x \in$ int $F$ for some $k$-face of $\mathscr{T}_{A}, k \leqq n$, (i.e. $F$ is a $k$-face of some $C \in \mathscr{T}_{A}$ ). We prove by descending induction on $k$, beginning from $k=n$, that if $x \in \operatorname{int} F$ for some $k$-face of $\mathscr{T}_{A}$, then

$$
\cup \mathscr{T}_{\boldsymbol{A}}
$$

contains a neighbourhood of $x$.

This is clear if $k=n$. Suppose then that $k=n-1$. Then $x \in \operatorname{int} F$ for some $F$ which is an $(n-1)$-face of some $C_{v} \in \mathscr{T}_{A}, v \in V_{A}$. Then there is a half-space $H$ of $H^{n}$ such that $H \supset C_{v}$ and that $\partial H \supset F$. Let $L$ be the hyperbolic line passing through $v$ which is orthogonal to $\partial H$. Let $R \subset L$ be the ray with endpoint $v$ such that $R \cap H$ is compact. If now $u \in R, u \neq v$, and if $r$ is so chosen that $\partial D(u, r) \supset F \cap A$, then

$$
D(u, r) \cap A_{v}=F \cap A_{v} .
$$

In view of (C9) and (C5), there is a first point $u$ on $R \backslash\{v\}$ such that for some $b \in A \backslash A_{v}$,

$$
d(u, b)=d(u, a)
$$

for $a \in F \cap A_{v}$. It follows that $u \in V_{A}, C_{u}$ has face $F$ and that $C_{v} \cup C_{u}$ contains a neighbourhood of $x$.

Suppose then that $x \in$ int $F$ for some $p$-face of $\mathscr{T}_{A}, p<n-1$, and that (C8) is true for $k>p$. Since $\mathscr{T}_{A}$ is locally finite, we can find $r>0$ such that if $F^{\prime}$ is a face of some $C \in \mathscr{T}_{A}$ and $D(x, r) \cap F^{\prime} \neq \emptyset$, then $x \in F^{\prime}$. Let $D^{\prime}=D(x, r) \backslash F$ which is a connected set since $p<n-1$. Our assumption on $r$ implies that if $y \in D^{\prime} \cap\left(\cup \mathscr{T}_{A}\right)$, then $y \in$ int $F^{\prime}$ for some $k$-face $F^{\prime}$ of $\mathscr{T}_{A}, k>p$. Hence the inductive assumption implies that $D^{\prime} \cap\left(\cup \mathscr{T}_{A}\right)$ is open in $D^{\prime}$. Since it is also closed in $D^{\prime}$ and evidently non-empty, we have the result that $D^{\prime} \subset \cup \mathscr{T}_{A}$. It follows that $D(x, r) \subset \cup \mathscr{T}_{A}$. Hence (C8) is true also for $p$. We have proved that $\cup \mathscr{T}_{A}$ is open. Our theorem is now completely proved.

Triangulations. So we now have a method to construct tessellations of $H^{n}$. We now make a triangulation out of the tessellation $\mathscr{T}_{A}$. A $k$-cell $S$ of $H^{n}$ is a (hyperbolic) $k$-simplex if it is of the form

$$
S=\operatorname{Co}\left(\left\{a_{0}, \ldots, a_{k}\right\}\right)
$$

for some $a_{i} \in H^{n}$. A family $\mathscr{K}$ of $n$-simplexes of $H^{n}$ is a triangulation of $H^{n}$ if it is a locally finite cover of $H^{n}$ and if for all $S, S^{\prime} \in \mathscr{K}$ the intersection 
is either empty or a common face. Note that faces of a simplex are also simplexes. We say that $S$ is a $k$-simplex of $\mathscr{K}$ if it is a $k$-face of some $n$-simplex of $\mathscr{K}$; vertices of $\mathscr{K}$ are 0 -simplexes of $\mathscr{K}$.

We now give a condition when we can form a triangulation $\mathscr{K}$ of $H^{n}$ whose set of vertices is a given set and which is, furthermore, $G$-invariant for some discrete Möbius group $G$ (i.e. $g(T) \in \mathscr{K}$ if $T \in \mathscr{K}$ and $g \in G$ ).

Theorem C2. Let $G$ be a discrete group of hyperbolic isometries of $H^{n}$ and let $A \subset H^{n}$ be a G-invariant set such that $A$ satisfies (C5) for some $M>0$. Furthermore, suppose that if $T$ is a cell of the tessellation $\mathscr{T}_{A}$ of Theorem $\mathrm{Cl}$ and $g \in G \backslash\{\mathrm{id}\}$, then

$$
\operatorname{T\cap } g(T)=\emptyset .
$$

Then there is a G-invariant triangulation $\mathscr{K}$ of $H^{n}$ whose set of vertices is $A$ such that if $T \in \mathscr{K}$ has vertices $a_{0}, \ldots, a_{n}$, then there is $v \in H^{n}$ for which

$$
d\left(v, a_{0}\right)=\ldots=d\left(v, a_{n}\right) \leqq d(v, a)
$$

when $a \in A$.

Proof. Since $A$ satisfies (C5), $\mathscr{T}_{A}$ is a locally finite tessellation of $H^{n}$. Obviously, the set $V_{A}$ is $G$-invariant if $A$ is and obviously then $A_{g(v)}=g\left(A_{v}\right)$ and $C_{g(v)}=g\left(C_{v}\right)$ for $g \in G$. It follows that $\mathscr{T}_{A}$ is also $G$-invariant. Hence we need only to subdivide the cells of $\mathscr{T}_{A}$ into simplexes in such a way that the number of vertices is not increased.

We first define a complete order $<$ in $A / G$; in view of countability of $G$ this is easily done. If $a \in A$, we denote

$$
\bar{a}=\text { class of } a \text { in } A / G .
$$

Finally, we denote by $\mathscr{T}_{k}$ the set of $k$-cells of $\mathscr{T}_{A}$.

The construction of $\mathscr{K}$ is inductive. We subdivide each $\mathscr{T}_{k}$ into $k$-simplexes, beginning from $k=0$, and then extend this subdivision to $\mathscr{T}_{k+1}$. We denote by $\mathscr{K}_{k}$ the subdivision $\mathscr{T}_{k}$ which we have obtained.

To obtain $\mathscr{T}_{0}$ and $\mathscr{T}_{1}$ we need do nothing: we simply set $\mathscr{K}_{0}=\mathscr{T}_{0}$ and $\mathscr{K}_{1}=\mathscr{T}_{1}$. Suppose then that we have constructed $\mathscr{K}_{k}$ for some $k \geqq 1$. We now construct $\mathscr{K}_{k+1}$. Pick $T \in \mathscr{T}_{k+1}$. Let $v_{1}, \ldots, v_{r}$ be its vertices. By (C11), we can denote them in such a way that

$$
\bar{v}_{1}<\ldots<\bar{v}_{r} .
$$

Let $\mathscr{K}_{T}=\left\{S \in \mathscr{K}_{k}: S \subset T\right.$ and $\left.v_{1} \notin S\right\}$ and let the elements of $\mathscr{K}_{T}$ be $S_{1}, \ldots, S_{q}$. Let

$$
S_{i}^{\prime}=\operatorname{Co}\left(S_{i} \cup\left\{v_{1}\right\}\right) .
$$

Then $S_{1}^{\prime}, \ldots S_{q}^{\prime}$ is a subdivision of $T$ into simplexes which extends the subdivision of $\partial T$. We do this for all $T \in \mathscr{T}_{k+1}$. 
In the $(n+1)$ th step we have $\mathscr{K}_{n}=\mathscr{K}$. It is a locally finite cover of $H^{n}$ since $\mathscr{T}_{A}$ is. Using (C4) one easily sees that $\mathscr{K}$ satisfies (C10). Hence $\mathscr{K}$ is a triangulation of $H^{n}$ which is $G$-invariant by construction since the order in (C13) is preserved if $T$ is replaced by $g(T)$ and $v_{i}$ by $g\left(v_{i}\right)$ for $g \in G$. Since we have not increased the number of vertices during the construction, the set of vertices of $\mathscr{K}$ is $A$. Finally, in view of $(\mathrm{C} 2)$ and $(\mathrm{C} 3)$, we have $(\mathrm{C} 12)$.

D. Triangulations with bounded flatness. In this section we construct triangulations of $H^{n}$ whose simplexes cannot be very flat. For this we need a measure for the flatness of a simplex. We will now give such a measure which may not be the most natural but is the most convenient one for this paper.

If $a=\left(a_{0}, \ldots, a_{k}\right) \in\left(H^{n}\right)^{k+1}$, let $V_{i}(a)$ be the hyperbolic subplane of $H^{n}$ of minimal dimension containing the points $a_{0}, \ldots, a_{i-1}$ and set

$$
\delta(a)=\min _{0<i \leqq k}\left(a_{i}, V_{i}(a)\right) .
$$

If the points $a_{i}$ are the vertices of a hyperbolic $k$-simplex $T$, we denote

$$
T=T(a)
$$

obviously this happens if and only if $\delta(a)>0$. Thus if $\delta(a)>0$, then also $\delta\left(a_{\sigma(0)}, \ldots, a_{\sigma(k)}\right)>0$ for any permutation $\sigma$ of the numbers $0, \ldots, k$. Thus setting

$$
F(T)=\min _{\sigma} d(T) / \delta\left(a_{\sigma(0)}, \ldots, a_{\sigma(k)}\right),
$$

the minimum taken over all permutations $\sigma$ of $0, \ldots, k$, we get a number $F(T) \in[1, \infty)$ and it seems evident that the bigger $F(T)$ is, the flatter $T$ is. So we call $F(T)$ the flatness of $T$.

We define the flatness $F(T)$ in the same manner also for euclidean simplexes $T$. We also denote $T=T(a)$ as in (D0) if the $(k+1)$-tuple of the vericices of $T$ is $a=\left(a_{0}, \ldots, a_{k}\right)$. If confusion is possible we say whether we mean euclidean or hyperbolic simplexes.

So we will now construct triangulations whose simplexes are of bounded flatness. Furthermore, the triangulations will be $G$-invariant under the action of a torsionless Möbius group $G$ of $H^{n}$. Given such a $G$ we define

$$
\begin{gathered}
\varrho(x)=\inf \{d(x, g(x)): g \in G \backslash\{\operatorname{id}\}\}>0, \text { and } \\
\mu(x)=\min (\varrho(x), 1),
\end{gathered}
$$

if $x \in H^{n}$ (if $G=\{$ id $\}$, we set $\varrho(x)=\mu(x)=1$ for all $x \in H^{n}$ ). Since $G$ is torsionless, $\mu(x)>0$ always. We observe the following relations for $\mu$ :

$$
\mu(x)-2 d(x, y) \leqq \mu(y) \leqq \mu(x)+2 d(x, y)
$$


for all $x, y \in H^{n}$, implying

$$
\frac{c-2}{c} \mu(x) \leqq \mu(y) \leqq \frac{c+2}{c} \mu(x)
$$

if $x, y \in H^{n}$ and $d(x, y) \leqq \mu(x) / c, c>0$.

Now we construct a $G$-invariant set $A \subset H^{n}$ such that every $x \in H^{n}$ is not very far from a point of $A$, measured with the number $\mu(x)$, but that if $a, b \in A$ are distinct, then $d(a, b)$ cannot be very small in comparison with $\mu(a)$ or $\mu(b)$. Then we will get a $G$-invariant triangulation of $H^{n}$ by the theorem of the preceding section and if we are careful in the construction of $A$, this will be the desired triangulation.

Let $D(x, r)$ be the closed hyperbolic disk with center $x \in H^{n}$ and radius $r>0$. If $a_{1}, \ldots, a_{k} \in H^{n}$, let $V\left(a_{1}, \ldots, a_{k}\right)$ be the hyperbolic plane of minimal dimension containing the points $a_{i}$.

When picking elements of $A$, we must make sure that the simplexes obtained by Theorem $\mathrm{C} 2$ are not very flat. This is accomplished by

Lemma D1. Let $x \in H^{n}$ and let $B \subset D(x, \mu(x) / 3)$ be a set such that $d(a, b) \geqq$ $\mu(a) / 40$ for all distinct $a, b \in B$. Then

(a) $B$ contains at most $q$ points for some $q=q(n)$, and

(b) there is an integer $p=p(n)$ such that if $r>0$ is given, there is $y \in D(x, r)$ for which

$$
d\left(y, V\left(b_{1}, \ldots, b_{j}\right)\right) \geqq r / 4^{p}
$$

for all $b_{1}, \ldots, b_{j} \in B$ such that the dimension of $V\left(b_{1}, \ldots, b_{j}\right)$ is less than $n$.

Proof of (a). By (D3), $\mu(b) \geqq \mu(x) / 3$ for all $b \in B$. Thus $d(a, b) \geqq \mu(x) / 120$ for all $a, b \in B, a \neq b$, and thus, if $m$ is the $n$-dimensional hyperbolic measure,

$$
\left.\sum_{b \in \boldsymbol{B}} m(D(b, \mu(x) / 240))\right) \leqq m(D(x, \mu(x))) .
$$

There are positive constants $c$ and $c^{\prime}$ (depending on $n$ ) such that $m(D(z, r)) \in\left[c r^{n}, c^{\prime} r^{n}\right]$ if $z \in H^{n}$ and $r \leqq 1$. Since $\mu(x) \leqq 1$, it follows that in $B$ there are at most $240^{n} c^{\prime} / c$ points, proving (a).

Let now $V_{1}, \ldots, V_{k}$ be the hyperbolic planes which are of dimension $<n$ and which are of the form $V\left(b_{1}, \ldots, b_{s}\right), b_{j} \in B$. Then, by (a), $k \leqq 2^{q}$ and thus the lemma is true for $p=2^{q}$ if we can show that, given $z \in H^{n}, s>0$ and $i \leqq k$, then there is $y \in D(z, s)$ such that $d\left(y, V_{j}\right) \geqq s / 4^{i}$ if $j \leqq i$.

We show this by induction on $i$. Obviously it is true for $i=0$. Assume that it is true for $i-1$. We prove it then for $i$. By the inductive assumption, there is $w \in D(z, r / 2)$ such that $d\left(w, V_{j}\right) \geqq r / 2 \cdot 4^{i-1}$ if $j \leqq i-1$. We can find $y \in D\left(w, r / 4^{i}\right)$ such that $d\left(y, V_{i}\right) \geqq r / 4^{i}$. But then $d\left(y, V_{j}\right) \geqq d\left(y, \partial D\left(w, r / 2 \cdot 4^{i-1}\right)\right) \geqq r / 4^{i}$ if $j \leqq i-1$. Since $y \in D(z, r)$, (b) follows. 
Now we can prove

Lemma D2. Let $G$ and $\mu$ be as above. Then there is a discrete set $A \subset H^{n}$ such that

(a) $g A=A$ for every $g \in G$,

(b) $d(x, A) \leqq \mu(x) / 10 \leqq 1 / 10$ for $x \in H^{n}$,

(c) $d(a, b) \geqq \mu(a) / 40$ for distinct $a, b \in A$, and

(d) there is a constant $M=M(n) \geqq 1$ such that if $a_{0}, \ldots, a_{k} \in A, k \leqq n$, and if $d\left(a_{i}, a_{j}\right) \leqq \mu\left(a_{j}\right) / 4, i, j \leqq k$, then $a_{0}, \ldots, a_{k}$ are the vertices of a hyperbolic $k$-simplex $T$ for which the flatness $F(T) \leqq M$.

Proof. Let $X=\left\{x_{1}, x_{2}, \ldots\right\} \subset H^{n}$ be a countable set which is dense in $H^{n}$. We define inductively sets $A_{0}, A_{1}, \ldots$ satisfying

(a') $A_{i}=A_{i-1}$ or $A_{i}=A_{i-1} \cup G a_{i}^{\prime}$ for some $a_{i}^{\prime} \in D\left(x_{i}, \mu\left(x_{i}\right) / 20\right) \quad\left(A_{-1}=\emptyset\right)$,

(b') $\left\{x_{1}, \ldots, x_{i}\right\} \subset \cup\left\{D(a, \mu(a) / 10): a \in A_{i}\right\}$,

(c') $d(a, b) \geqq \mu(a) / 40$ for distinct $a, b \in A_{i}$, and

$\left(\mathrm{d}^{\prime}\right)$ there is $c=c(n)>0$ such that if $a \in A_{i} \backslash A_{i-1}$ and $a_{1}, \ldots, a_{k} \in$ $A_{i-1} \cap D(a, \mu(a) / 4), k \leqq n$, then $d\left(a, V\left(a_{1}, \ldots, a_{k}\right)\right) \geqq c \mu(a)$.

We set $A_{0}=\emptyset$ which obviously satisfies conditions $\left(\mathrm{a}^{\prime}\right)-\left(\mathrm{d}^{\prime}\right)$. Assume that $A_{i-1}$ satisfying these conditions has been constructed. If $d\left(x_{i}, a\right) \leqq \mu(a) / 10$ for some $a \in A_{i-1}$, we sêt $A_{i}=A_{i-1}$. Obviously $\left(\mathrm{a}^{\prime}\right)-\left(\mathrm{d}^{\prime}\right)$ are valid in this case. Otherwise there is by Lemma D1 and (D3) $a_{i}^{\prime} \in D\left(x_{i}, \mu\left(x_{i}\right) / 20\right)$ such that (d') is true if $a=a_{i}^{\prime}$ for some $c=c(n)$. We set $A_{i}=A_{i-1} \cup G a_{i}^{\prime}$. Conditions $\left(\mathrm{a}^{\prime}\right),\left(\mathrm{b}^{\prime}\right)$ and $\left(\mathrm{d}^{\prime}\right)$ are now easily seen to be true using (D3) and the fact that $G a \cap$ int $D(a, \mu(a))=\{a\}$. To show that it satisfies $\left(\mathrm{c}^{\prime}\right)$, we can reason as follows.

It suffices to show that if $a \in A_{i-1}$, then

(i) $d\left(a, a_{i}^{\prime}\right) \geqq \mu(a) / 30$, and

(ii) $d\left(a, a_{i}^{\prime}\right) \geqq \mu\left(a_{i}^{\prime}\right) / 40$,

since the other cases follow from these, from the inductive assumption and from the $G$-invariance of $\mu$.

Assume that (i) is not true. Then by (D3), $\mu\left(a_{i}^{\prime}\right) \leqq 32 \mu(a) / 30$. Similarly, since $d\left(x_{i}, a_{i}^{\prime}\right) \leqq \mu\left(x_{i}\right) / 20, \mu\left(x_{i}\right) \leqq 20 \mu\left(a_{i}^{\prime}\right) / 18$. Thus $\mu\left(x_{i}\right) \leqq 640 \mu(a) / 540=32 \mu(a) / 27$. Now, $d\left(a, x_{i}\right) \leqq d\left(a, a_{i}^{\prime}\right)+d\left(a_{i}^{\prime}, x_{i}\right) \leqq \mu(a) / 30+\mu\left(x_{i}\right) / 20 \leqq(1 / 30+32 / 540) \mu(a)=(1 / 3+16 / 27) \cdot$ $\mu(a) / 10<\mu(a) / 10$, a contradiction since $d\left(a, x_{i}\right)>\mu(a) / 10$. Thus (i) is true.

This implies then (ii), since if $d\left(a, a_{i}^{\prime}\right)<\mu\left(a_{i}^{\prime}\right) / 40$, we get by (D3), $\mu\left(a_{i}^{\prime}\right)<$ $40 \mu(a) / 38$. Thus $d\left(a, a_{i}^{\prime}\right) \leqq \mu\left(a_{i}^{\prime}\right) / 40<40 \mu(a) / 38 \cdot 40 \leqq \mu(a) / 38$, a contradiction with (i) and we have also (ii).

Thus we have sets $A_{0} \subset A_{1} \subset \ldots$ and we set $A=\bigcup_{i \geqq 0} A_{i}$. We show that Lemma $\mathrm{D} 2$ is true with this $A$. Cases (a) and (c) are obvious by (a') and (c'). Then (c) together with (D3) implies that $A$ is discrete. To prove (b), observe that the discreteness of $A$ implies that $\{D(a, \mu(a) / 10): a \in A\}$ is locally finite. Thus

$$
X^{\prime}=\cup\{D(a, \mu(a) / 10): a \in A\}
$$


is closed. Since $X^{\prime} \supset X$ and $X$ is dense in $H^{n}$, we must have $X^{\prime}=H^{n}$. This implies (b) since, in addition, $\mu(x) \leqq 1$.

Finally, we prove (d). Let $a_{0}, \ldots, a_{k}$ be as in (d). Since $d\left(a_{i}, a_{j}\right) \leqq \mu\left(a_{j}\right) / 4$, no two points in the sequence $a_{0}, \ldots, a_{k}$ can be $G$-equivalent by the definition of $\mu$. Thus if $j(i)$ is the number such that $a_{i} \in A_{j(i)} \backslash A_{j(i)-1}$, then all the numbers in the sequence $j(0), \ldots, j(k)$ are distinct. Let $\sigma$ be the permutation of the numbers $0, \ldots, k$ such that the sequence $j(\sigma(0)), \ldots, j(\sigma(k))$ is increasing. Let $b_{i}=a_{j(\sigma(i))}$. Then, by $\left(\mathrm{d}^{\prime}\right)$,

$$
d\left(b_{i}, V\left(b_{0}, \ldots, b_{i-1}\right)\right) \geqq c \mu\left(b_{i}\right)>0
$$

for all $i \geqq 0$. Thus $b_{0}, \ldots, b_{k}$ are the vertices of a $k$-simplex $T$. We have $d(T) \leqq$ $\mu\left(b_{0}\right) / 2$. By (D3), $\mu\left(b_{i}\right) \geqq \mu\left(b_{0}\right) / 2$. Thus the flatness $F(T) \leqq c$ and the lemma is proved.

We now need only to apply Theorem $\mathrm{C} 2$ to the set $A$ constructed above to get the theorem on triangulations at which we have been aiming:

Theorem D. Let $G$ be a torsionless discrete Möbius group of $H^{n}$. Then there is a G-invariant triangulation $\mathscr{K}$ of $H^{n}$ such that

(a) $d(T) \leqq 1$ for all $T \in \mathscr{K}$,

(b) $g(T) \cap T=\emptyset$ for all $g \in G \backslash\{$ id $\}$ and $T \in \mathscr{K}$,

(c) there is a number $M=M(n) \geqq 1$ such that for every $T \in \mathscr{K}$ the flatness $(c f .(\mathrm{D} 1))$

$$
F(T) \leqq M, \text { and }
$$

(d) there is an integer $N=N(n)$ such that the number of simplexes of $\mathscr{K}$ with a common vertex does not exceed $N$.

Proof. Let $A \subset H^{n}$ be a discrete $G$-invariant set as in Lemma D2. Let $\mathscr{K}$ be the triangulation of $H^{n}$ whose set of vertices is $A$ which is given by Theorem C2. We claim that $\mathscr{K}$ satisfies conditions (a)-(d).

Let $T \in \mathscr{K}$ be a simplex which has vertices $a_{0}, \ldots, a_{k} \in A$. By Theorem $\mathrm{C} 2$, there are $v \in H^{n}$ and $r>0$ such that $d\left(v, a_{i}\right)=r$ for all $i$ and that $d(v, a) \geqq r$ for all $a \in A$. Since $d(x, A) \leqq \mu(x) / 10$ for all $x \in H^{n}$ by Lemma D2 (b), we have $r \leqq$ $\mu(v) / 10$. Hence

$$
d(T) \leqq 2 \mu(v) / 10 \leqq 1
$$

and we have (a). By the definition of $\mu$, then $d(T, g(T)) \geqq \mu(v)-2 r \geqq 0.8 \mu(v)>0$ for all $g \in G \backslash\{$ id $\}$, which implies (b).

By (D3), $\mu(a) \geqq(10 / 8) \mu(v)$. Hence if $i \neq j, \quad d\left(a_{i}, a_{j}\right) \leqq(2 / 10) \mu(v) \leqq \mu\left(a_{i}\right) / 4$. Then Lemma D2 (d) implies that indeed $F(T) \leqq M=M(n)$ and (c) is true. Finally, (d) follows from Lemma D1 (a) and the theorem is proved.

Remark. Actually, if $x \in T \in \mathscr{K}$, we have the following, more precise, estimate for $d(T)$

$$
\mu(x) / 80 \leqq d(T) \leqq \mu(x) / 4 .
$$


Indeed, since $d(x, v) \leqq \mu(v) / 10$, (D3) and (D6) imply the second inequality and then it, (D3) and Lemma D2 (c) imply the first one.

E. A canonical map between simplexes. We now define a canonical map between two $n$-simplexes $T$ and $S$. The simplexes may be either hyperbolic or euclidean (independently) and the map depends on a given order of vertices. If both simplexes are euclidean, then the map will be affine.

Let $T=T(a)$ and $S=T(b), a=\left(a_{0}, \ldots, a_{n}\right), b=\left(b_{0}, \ldots, b_{n}\right)$ be two $n$-simplexes where the notation is as in (D0). We will now define a map $h=h_{a b}: T \rightarrow S$ which extends the map $a_{i} \mapsto b_{i}$ of vertices. Let $T_{k}$ and $S_{k}$ be the union of $k$-simplexes of $T$ and $S$, respectively. We define the map inductively: first in $T_{0}$, then in $T_{1}$, etc. We define $h$ in $T_{0}$ by

$$
h\left(a_{i}\right)=b_{i}
$$

Suppose then that $h$ has been defined in $T_{k-1}$. We extend $h$ to $T_{k}$ as follows. Let $T^{\prime}=T\left(a_{i_{0}}, \ldots, a_{i_{k}}\right), i_{0}<\ldots<i_{k}$, be a $k$-subface of $T$ and let $T^{\prime \prime}$ be the $(k-1)$-subface of $T^{\prime}$ opposite to $a_{i_{0}}$. Then $h$ is already defined in $T^{\prime \prime}$. If $y \in T^{\prime \prime}$, let $s_{y}$ be the (hyperbolic or euclidean) segment joining $a_{i_{0}}$ and $y$; similarly, let $s_{y}^{\prime}$ be the segment joining $h(y)$ and $b_{i_{0}}=h\left(a_{i_{0}}\right)$. We now define $h$ in $T^{\prime}$ by the requirements that

$$
h\left(s_{y}\right)=s_{y}^{\prime}
$$

and that $h \mid s_{y}$ is an affine stretch in the metrics involved (which may be hyperbolic or euclidean independently).

We record here some properties of the map $h_{a b}$. If the $(n+1)$-tuples $a$ and $b$ are as above, let $a^{\prime}=\left(a_{i_{0}}, \ldots, a_{i_{k}}\right)$ and $b^{\prime}=\left(b_{i_{0}}, \ldots, b_{i_{k}}\right)$ be $k$-subfaces of $T(a)$ and $T(b)$ where $i_{0}<\ldots<i_{k}$. Then obviously

$$
h_{a^{\prime} b^{\prime}}=h_{a b} \mid T\left(a^{\prime}\right) \text {. }
$$

Also, if $c$ is another $(n+1)$-tuple, we have the following composition rule:

$$
h_{a c}=h_{b c} \circ h_{a b} \text {. }
$$

Finally, if $\alpha$ and $\beta$ are euclidean similarities or hyperbolic isometries (depending on whether the simplexes are euclidean or hyperbolic), then

$$
h_{\alpha(a) \beta(b)}=\beta \circ h_{a b} \circ\left(\alpha^{-1} \mid T(\alpha(a))\right) .
$$

We also need to know that $h_{a b}$ is always a fairly regular map. It is always quasiconformal, even bilipschtiz; for explicit calculations in case $n=2$, see [5, Lemma 4.1]. We now show that if the simplexes $T=T(a)$ and $S=T(b)$ vary in such a way that they satisfy a boundedness condition involving flatness (see the preceding section), then the maps $h_{a b}$ are uniformly $K$-quasiconformal for some $K$.

Lemma E. Let $n \geqq 2$ and $M \geqq 1$. Then there is $K=K(n, M)$ with the following property. Let $T=T(a)$ and $S=T(b)$ be two (hyperbolic or euclidean) simplexes such that $F(T) \leqq M$ and $F(S) \leqq M$. If $T$ or $S$ is hyperbolic, assume in addition 
that $d(T) \leqq M$ or $d(S) \leqq M$, respectively. Then the map $h_{a b}: T \rightarrow S$ is $K$-quasiconformal.

Proof. Suppose first that both $T$ and $S$ are euclidean. If there is no such $K$ for which the lemma is true, we can find sequences $T_{i}=T\left(a_{i}\right)$ and $S_{i}=T\left(b_{i}\right)$, where $a_{i}=\left(a_{i 0}, \ldots, a_{i n}\right)$ and $b_{i}=\left(b_{i 0}, \ldots, b_{i n}\right)$, of euclidean $n$-simplexes such that

$$
F\left(T_{i}\right) \leqq M \quad \text { and } \quad F\left(S_{i}\right) \leqq M
$$

and such that the maps $h_{i}=h_{a_{i} b_{i}}$ have dilatation $K_{i} \rightarrow \infty$ as $i \rightarrow \infty$. Composing with auxiliary similarity maps, $\mathrm{cf}$. (E3), which change neither flatness nor dilatation, we can assume that $a_{i 0}=b_{i 0}=0$ and that $d\left(T_{i}\right)=d\left(S_{i}\right)=1$. In view of this normalization, we can pass to a subsequence in such a way that, as $i \rightarrow \infty$,

$$
\begin{aligned}
& a_{i j} \rightarrow a_{j}^{\prime} \in R^{n} \quad \text { and } \\
& b_{i j} \rightarrow b_{j}^{\prime} \in R^{n} .
\end{aligned}
$$

Condition (E4) implies that $a^{\prime}=\left(a_{0}^{\prime}, \ldots, a_{n}^{\prime}\right)$ and $b^{\prime}=\left(b_{0}^{\prime}, \ldots, b_{n}^{\prime}\right)$ are the vertices of non-degenerate $n$-simplexes $T^{\prime}=T\left(a^{\prime}\right)$ and $S^{\prime}=T\left(b^{\prime}\right)$ (with flatness $\leqq M$ ). Let $K$ be the dilatation of $h^{\prime}=h_{a^{\prime} b^{\prime}}$. Now the maps $h^{\prime}$ and $h_{i}$ are affine maps. Hence we must have by (E5) that $K_{i} \rightarrow K<\infty$, a contradiction.

Suppose then that one (or both) of the simplexes are hyperbolic. Suppose, say, that $T$ is hyperbolic and $S$ euclidean. If the lemma is not true, we can find sequences $T_{i}$ and $S_{i}$ as above. We cannot now normalize to have $d\left(T_{i}\right)=1$ but we can still have that $a_{i 0}=e_{n}=(0, \ldots, 0,1)$. Again, we can pass to a subsequence in such a way that (E5) is true and that $d=\lim _{i \rightarrow \infty} d\left(T_{i}\right) \in[0, \infty)$ exists. If $d>0$, we obtain a contradiction as above. If $d=0$, we obtain a contradiction using the fact that hyperbolic geometry is infinitesimally euclidean. This means that, taking account of the bounded flatness, there is a non-degenerate euclidean simplex $T^{\prime}=T^{\prime}\left(a^{\prime}\right)$ such that the maps $h_{a^{\prime} a_{i}}$ are $K_{i}$-quasiconformal where $K_{i} \rightarrow 1$. Since the map $h_{a^{\prime} b^{\prime}}$ is $K$-quasiconformal for some $K<\infty$, a contradiction follows (cf. (E2)).

F. The proof of the main theorem. We now apply the preceding results and construct the $G$-automorphic mapping whose existence was claimed in Theorem A.

Let $\mathscr{K}$ be the $G$-invariant triangulation of $H^{n}$ whose set of vertices is $A$ and which is given by Theorem $\mathrm{D}$. We then choose points $a_{i} \in A, i<k \leqq \infty$, such that every orbit $G a, a \in A$, contains exactly one point $a_{i}$.

Then let the integer $N$ be as in Theorem D (d). Let $B \subset R^{n}$ be a set containing $n N+1$ points in general position, i.e. any distinct $n+1$ points $b_{0}, \ldots, b_{n} \in B$ are the vertices of a non-degenerate euclidean $n$-simplex.

The first step in the construction of the $G$-automorphic map $f$ is to find a $G$ invariant map $\varphi: A \rightarrow B$ which is injective on the vertices of a triangle:

Lemma F. There is a G-invariant map $\varphi: A \rightarrow B$ such that if $a, b \in A, a \neq b$, are vertices in a simplex of $\mathscr{K}$, then $\varphi(a) \neq \varphi(b)$. 
Proof. It suffices to define $\varphi$ at the points $a_{i}$. Set $\varphi\left(a_{1}\right)=b$ where $b$ is an arbitrary point of $B$. Assume then that $\varphi\left(a_{i}\right)$ has been defined for $i \leqq p-1$. Let $b_{1}, \ldots$, $b_{q} \in A$ be the points of $A$ such that $b_{i}$ and $a_{p}$ are vertices in a simplex of $\mathscr{K}$ and such that $b_{i}=g_{i}\left(a_{j(i)}\right)$ for some $g_{i} \in G$ and $j(i)<p$. By Theorem $\mathrm{D}(\mathrm{d}), q \leqq n N$. Thus there is $b \in B \backslash\left\{\varphi\left(a_{j(i)}\right): i \leqq q\right\}$. We set $\varphi\left(a_{p}\right)=b$. This defines a unique $G$-invariant map $\varphi: A \rightarrow B$ which is the required map. Note that by Theorem D (b), $a$ and $g(a)$ are not vertices in a simplex $T \in \mathscr{K}$ for $a \in A$ and $g \in G \backslash\{$ id $\}$.

Let $B^{\prime}=\left\{\left(b_{0}, \ldots, b_{n}\right) \in B^{n+1}: b_{i}\right.$ distinct $\}$. Then every $b \in B^{\prime}$ is the $(n+1)$-tuple of a euclidean $n$-simplex $T_{b}=T(b)$, using the notation of (D0). We fix for every $b \in B^{\prime}$ a quasiconformal reflection $r_{b}: \bar{R}^{n} \rightarrow \bar{R}^{n}$ on $\partial T_{b}$, i.e. $r_{b}$ is an orientation reversing quasiconformal map interchanging the components of $\bar{R}^{n} \backslash \partial T_{b}$ such that

$$
r_{b} \mid \partial T_{b}=\mathrm{id}
$$

Note that $T_{b}$ depends only on the set $\left\{b_{0}, \ldots, b_{n}\right\}$ and we can assume that this is true of $r_{b}$, too.

We can now define a $G$-invariant map $f: H^{n} \rightarrow \bar{R}^{n}$ as follows. If $T \in \mathscr{K}$ is an $n$-simplex, let $a_{0}^{T}, \ldots, a_{n}^{T}$ be the vertices of $T$, enumerated in such a way that $a_{i}^{T}=$ $g_{i}^{T}\left(a_{j(i)}\right)$ for some $g_{i}^{T} \in G$ and $j(i) \geqq 1$ such that $j(0)<\ldots<j(n)$. By Theorem $\mathrm{D}(\mathrm{b})$, all the numbers $j(i)$ in the sequence are indeed distinct. Then we set, if $a=\left(a_{0}^{T}, \ldots, a_{n}^{T}\right)$ and $\varphi(a)=\left(\varphi\left(a_{0}^{T}\right), \ldots, \varphi\left(a_{n}^{T}\right)\right)$,

$$
f \mid T=h_{a \varphi(a)},
$$

where $h_{a \varphi(a)}: T \rightarrow T_{\varphi(a)}$ is the map defined in Section E, provided that $h_{a \varphi(a)}$ is orientation preserving. If this is not the case, we set

$$
f \mid T=r_{\varphi(a)} \circ h_{a \varphi(a)} .
$$

By (F1) and (E1), we get a well-defined continuous map $f: H^{n} \rightarrow \bar{R}^{n}$. Thus $f \mid T$ is always orientation preserving.

We show that $f$ is $G$-invariant. Let $T \in \mathscr{K}$ be as above and pick $g \in G$. Let $S=g(T)$. Then $a_{i}^{S}=g g_{i}^{T}\left(a_{j(i)}\right)$ and hence (E3) implies that $(f \mid S) \circ g=f \mid T$ since every $g \in G$ is orientation preserving.

Next we show that $g$ is $K$-quasimeromorphic for some $K=K(n)$. Observe first that the set $B^{\prime}$ is a finite set depending only on $n$. Thus the maps $r_{b}, b \in B^{\prime}$, are $K_{1}$-quasiconformal for some $K_{1}=K_{1}(n)$. For the same reason, the flatness $F\left(T_{b}\right) \leqq$ $M_{1}$ for some $M_{1}=M_{1}(n)$ if $b \in B^{\prime}$. Now Theorem $\mathrm{D}(\mathrm{c})$ and Lemma E imply that the maps $h_{a \varphi(a)}$ in (F2) and (F3) are $K_{2}$-quasiconformal for some $K_{2}=K_{2}(n)$. Thus, if $K=K_{1} K_{2}=K(n), f \mid T$ is $K$-quasiconformal for every $T \in \mathscr{K}$. It follows that $f$ is $\mathrm{ACL}^{n}$, and since $f \mid T$ is orientation preserving by the above definition for every $T \in \mathscr{K}$, it follows that $f$ is $K$-quasimeromorphic.

Finally, Theorem D (d) implies that $i(x, f) \leqq N$ for some $N=N(n)$. Theorem A is proved. 


\title{
References
}

[1] AleXander, J. W.: Note on Riemann spaces. - Bull. Amer. Math. Soc. 26, 1919-1920, 370-372.

[2] Beardon, A. F.: The geometry of discrete groups. - Graduate Texts in Mathematics 91. Springer-Verlag, New York-Berlin-Heidelkerg, 1983.

[3] Martio, O., and U. SRebro: Automorphic quasimeromorphic mappings in $\boldsymbol{R}^{n}$. - Acta Math. $135,1975,221-247$.

[4] Martio, O., and U. SRebro: On the existence of automorphic quasimeromorphic mappings in $R^{n}$. - Ann. Acad. Sci. Fenn. Ser. A I Math. 3, 1977, 123-130.

[5] TukiA, P.: On infinite dimensional Teichmüller spaces. - Ibid. 3, 1977, 343-372.

[6] Tukia, P.: Quasiconformal extension of quasisymmetric mappings compatible with a Möbius group. - Acta Math. 154, 1985, 153-193.

\author{
University of Helsinki \\ D'epartment of Mathematics \\ SF-00100 Helsinki \\ Finland
}

Received 14 May 1984 